Marquette University

e-Publications@Marquette

7-1-2013

Naturalizing Moral Justification: Rethinking the Method of Moral Epistemology

Theresa Weynand Tobin

Marquette University, theresa.tobin@marquette.edu

Alison Jaggar

University of Colorado - Bolder

Accepted version. Metaphilosophy, Vol. 44, No. 4 (July 2013): 409-439. DOI. (C) 2013 John Wiley \& Sons, Inc. Used with permission. 
Marquette University

\section{e-Publications@Marquette}

\section{Philosophy Faculty Research and Publications/Department of Philosophy}

This paper is NOT THE PUBLISHED VERSION; but the author's final, peer-reviewed manuscript. The published version may be accessed by following the link in the citation below.

Metaphilosophy, Vol. 44, No. 4 (July, 2013): 409-439. DOI. This article is (C) Wiley and permission has been granted for this version to appear in e-Publications@Marquette. Wiley does not grant permission for this article to be further copied/distributed or hosted elsewhere without the express permission from Wiley.

\section{Contents}

Abstract.

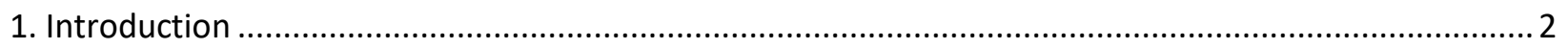

2. Why Do We Need a New Method for Doing Moral Epistemology? .................................................... 5

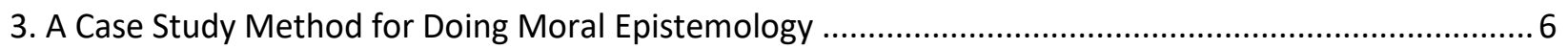

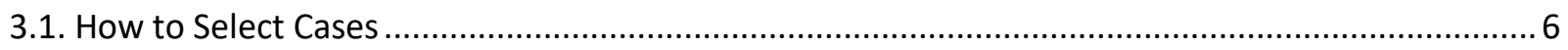

3.2. Describing, Analyzing, and Evaluating Practices of Moral Justification in Each Case ...................... 8

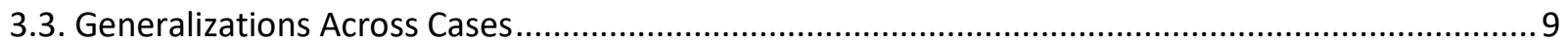

4. A Case Study: Moral Reasoning About FGC Among the Maasai......................................................... 9

4.1. Situating the Moral Dispute and Introducing the Disputants....................................................... 9

4.2. Evaluating Maasai FGC by Appealing to Women's Human Rights .............................................. 10

4.3. Evaluating Maasai FGC Through the Frame of Colonial History ................................................. 12

5. Assessing the Alternative Practices of Moral Justification Used in This Case...................................... 15

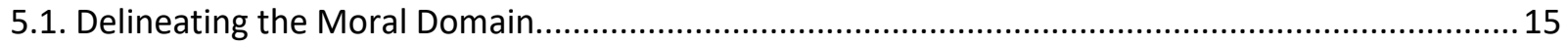

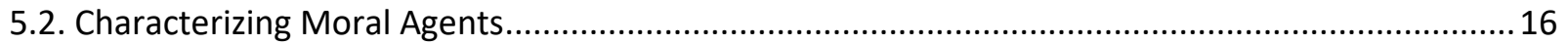

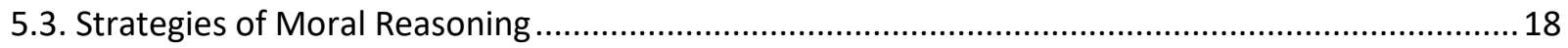

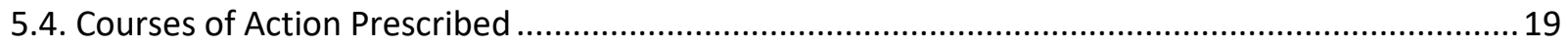

5.5. Summing Up Our Assessment of These Practices of Moral Justification........................................19 


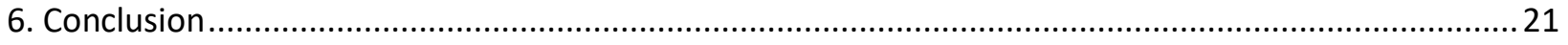

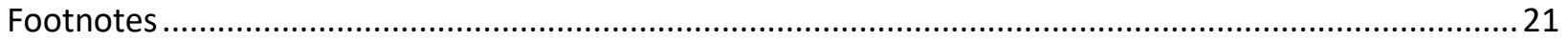

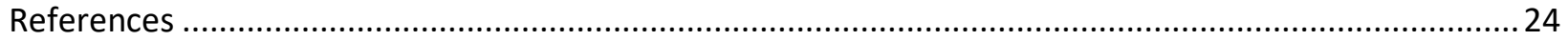

\title{
Naturalizing Moral Justification: Rethinking the Method of Moral Epistemology
}

\author{
Theresa W. Tobin
}

Department of Philosophy, Marquette University, Milwaukee, WI

Alison M. Jaggar

Department of Philosophy, University of Colorado at Boulder, Boulder, CO

\section{Abstract}

The companion piece to this article, "Situating Moral Justification," challenges the idea that moral epistemology's mission is to establish a single, all-purpose reasoning strategy for moral justification because no reasoning practice can be expected to deliver authoritative moral conclusions in all social contexts. The present article argues that rethinking the mission of moral epistemology requires rethinking its method as well. Philosophers cannot learn which reasoning practices are suitable to use in particular contexts exclusively by exploring logical relations among concepts. Instead, in order to understand which reasoning practices are capable of justifying moral claims in different types of contexts, we need to study empirically the relationships between reasoning practices and the contexts in which they are used. The article proposes that philosophers investigate case studies of real-world moral disputes in which people lack shared cultural assumptions and/or are unequal in social power. It motivates and explains the proposed case study method and illustrates the philosophical value of this method through a case study.

\section{Introduction}

Moral disagreement among people who are culturally or religiously diverse, or who stand to each other in relations of social inequality, is often intractable. People from widely diverse backgrounds often lack enough shared values or assumptions to establish intelligible communication, and social inequality among people makes domination and repression all too easy, even for the well-intentioned. We are interested in understanding how people can rationally resolve moral disagreements in situations where finding shared assumptions may be strained, or when parties to a dispute are unequal in social power and vulnerability. In this article, we prepare the ground for answering this urgent practical question by articulating and defending a method for doing moral epistemology designed to improve philosophical understanding of moral justification under conditions of diversity and inequality. 
Much existing philosophical work on moral justification occurs at a very high level of abstraction and sets quite bold theoretical goals. Many philosophers have assumed that moral justification can be pared down to a single reasoning practice or set of practices, which can be used to justify moral claims in any context. They therefore take the mission of moral epistemology to be the discovery or construction of a single, multipurpose model of moral reasoning. Philosophers have typically pursued this mission using "armchair" philosophical methods. They imagine what moral reasoning should be like either by constructing fictitious models of justification or by conceptualizing the logical constraints of moral reasoning under ideal conditions. In our view, the fruits of this philosophical labor as well as this way of laboring - that is, of doing moral epistemology-are inadequate for understanding how justified moral claims can be established in situations marked by cultural diversity and power inequality.

An analogy between medical research and moral epistemology is instructive here to introduce our point. A drug tested successfully within the controlled conditions of a clinical trial may or may not achieve the expected health outcome when used in the real world. In the context of real life, it might even prove harmful, depending on how well or poorly the conditions of real life approximate the conditions of the trial. However, "the" conditions of real life vary considerably. Clinical trials conducted in the 1990s in the United States of the HIV drug AZT delivered very promising results in that context, but this same drug regimen proved ineffective and potentially harmful when introduced to populations in sub-Saharan Africa that were struggling with epidemic levels of dehydration, malaria, and malnutrition, as well as a lack of sufficient health care infrastructure to support the complicated AZT regimen. There are enough physiological similarities among human beings to predict that when human beings are similarly situated they are likely to respond similarly to a given medical intervention; however, we are not all similarly situated. The controlled features of the clinical trial in the United States were designed for a population situated very differently from many populations in sub-Saharan Africa, rendering the results of the U.S. trial inapplicable in the African context.

Similarly, a model of moral justification developed under the controlled conditions of the philosopher's imagination and relying on his or her acknowledged or unacknowledged assumptions may or may not be capable of justifying moral claims under the conditions of real life. This may be not because the model has been applied incorrectly or unfairly but because it has been developed assuming a context with one set of features and then prescribed for all contexts, some of which have features that do not come close to approximating the features assumed in the philosopher's imagination. For example, the controlled conditions of philosophical imagination have tended to assume conditions in which interlocutors exchanging reasons have equal social power, and so philosophers have developed models of moral justification based on this assumption. These models may work well in situations where people are social equals, just as the AZT drug regimen worked well in populations who had adequate infrastructure and their basic health needs met. Yet a model of moral justification designed for conditions of social equality may be ineffective in situations where people have unequal social power, and may even be harmful if the model obscures power dynamics operative in that context or makes it too easy to rationalize power abuse. If the conditions of the real world diverge too much from the conditions of the philosopher's imagination, then prescribing a particular model of justification for real-world conditions may be like prescribing the AZT drug regimen in sub-Saharan contexts-both ineffective and potentially harmful.

The analogy between clinical research and research in moral epistemology is admittedly imperfect, but it is good enough to extract the point we wish to make: in order to develop appropriate medical 
interventions for specific types of contexts, clinical researchers have to examine carefully the relationship between drug protocols and the context in which they are to be used. Researchers cannot know whether a particular drug will be effective or harmful in a particular context without careful attention to the interaction between the two, because certain features of the context, including social, environmental, and cultural factors, partially determine which drug protocol is likely to be effective. Similarly, we suggest that justifying moral claims involves a relationship between a reasoning practice and the context in which it is used. Philosophers cannot know which practices are fitting to use in which types of situation without carefully studying the relationship between reasoning practices and their contexts of use, since certain features of a context partially determine which reasoning practices are capable of justifying moral claims in that context.

These considerations suggest two points about moral epistemology. First, and as we argue in the companion piece to this article, "Situating Moral Justification," we think that moral epistemology should adopt a more modest mission. Rather than searching for the single, multipurpose model of reasoning capable of justifying moral claims in all contexts, our mission instead should be to seek guidelines enabling reasoners to select reasoning practices likely to be effective in particular types of situations. Second, we think the study of moral justification needs to be much more empirically grounded. In order to understand which reasoning practices are capable of justifying moral claims in different types of contexts, we need to study empirically the relationships between reasoning practices and the contexts in which they work well.

Like a number of other contemporary philosophers, we advocate a methodological turn in moral and political philosophy, including moral epistemology, toward more empirically informed philosophical research. This turn is often called a move toward naturalizing epistemology and methodology because philosophers are attempting to make their research more continuous with results from empirical science. Some philosophers are using empirical findings from other disciplines, whereas other philosophers are attempting to conduct their own empirical research. Our proposal shares in the naturalizing spirit of these developments, but it suggests a new and distinct method for doing empirically grounded-naturalized-moral epistemology.

Specifically, we propose that philosophers investigate case studies of real-world moral disputes in which people lack shared cultural assumptions and/or are unequal in social power. Case studies always focus on what is being studied in relation to its environment, which makes this a promising method for investigating relationships between reasoning practices and their contexts of use. When investigating a case study, philosophers identify and explain the reasoning people are actually using in their particular attempts to justify a moral claim, and evaluate whether or not those reasoning practices are likely to facilitate or undermine moral justification in that case. We argue that working through case studies enables philosophers to understand better the obstacles to achieving moral justification that diversity and social power inequality actually pose, as opposed to the obstacles philosophers imagine they pose. Focusing on case studies may also enable philosophers to identify reasoning practices that actually work to mitigate the disruptive impact of these obstacles in certain cases, rather than the practices philosophers imagine to work.

This article motivates and explains our proposed case study method in moral epistemology and goes on to defend and illustrate the philosophical value of this method. Section 2 motivates our proposal for using case studies in moral epistemology, making reference to our critical work in the first of these 
companion articles. In section 3, we explain what a case study is and our proposal for how to use this method in moral epistemology. The remainder of the article defends and illustrates our proposal by sharing one of our case studies, demonstrating how we analyze and assess it.

\section{Why Do We Need a New Method for Doing Moral Epistemology?}

In "Situating Moral Justification" we challenged the idea that moral epistemology's mission is to establish a single, all-purpose reasoning strategy for moral justification, and we illustrated this claim through a critical assessment of discourse ethics. We argued that using the reasoning recommended by discourse ethics is not rational in all circumstances. Specifically, we argued that it is unlikely to be rational in situations marked by cultural diversity and social inequality because the reasoning strategy is too susceptible to misuse by those with more social power. We used the example of discourse ethics to support our larger point, which is that no reasoning practice can be assumed to deliver authoritative moral conclusions independently of the social context in which it is used.

In our view, most philosophers have underestimated the challenges that diversity and inequality can pose to achieving moral justification in real-world settings. To be minimally acceptable, a reasoning practice must be plausible to and usable by moral agents in the case at hand, nonabusive of social power or vulnerability, and capable of delivering feasible conclusions. We take these conditions partially to define a perspective from which to validate moral claims in the types of contexts with which we are concerned. They provide some initial, broadly normative constraints on reasoning strategies that may count as minimally acceptable, and they guide our evaluation of reasoning practices in the real-world cases of moral argumentation that we study. We contend, however, that what it means to satisfy these conditions will depend on the situation in which the occasion for moral justification arises. The situational features that influence whether and how a reasoning practice will satisfy these adequacy conditions include the social relations of power and vulnerability among moral interlocutors in particular situations of dispute, as well as the particular moral vocabularies and styles of reasoning that are available, meaningful, and usable to and by various parties.

Taking seriously the view that moral justification always requires situated, social reasoning practices suggests the need for a new mission for the part of moral epistemology that studies moral justification. The mission can no longer be to establish a single reasoning practice or set of practices for use in any and all situations. This mission might be suitable for a world in which diversity and inequality were not ubiquitous, but this is not our world. In our world, to prescribe for all contexts a single practice of justification that has been developed under the controlled conditions of the philosopher's imagination may at best be ineffective and at worst harmful (potentially reinforcing or rationalizing power abuse) in situations that do not match these conditions.

Rethinking the mission of moral epistemology requires rethinking its method as well. Philosophers cannot understand which reasoning practices are suitable to use in which contexts exclusively by imagining or exploring logical relations among concepts. Instead, in order to understand which reasoning practices and argumentative strategies are capable of justifying moral claims in different types of contexts, we need to study empirically the relationships between reasoning practices and the contexts in which they are used.

We propose that philosophers investigate case studies of real-world moral disputes in which people lack shared cultural assumptions and/or are unequal in social power. A case study model of naturalizing is 
particularly well suited for the conception of moral justification we defend as a situated social practice. Through investigating case studies, philosophers can scrutinize the details of particular situations in order to discern which social variables are salient in given cases, and examine how those variables operate either to support or undermine reasoning that is plausible, usable, power sensitive, and capable of delivering feasible conclusions.

\section{A Case Study Method for Doing Moral Epistemology}

A case study is a detailed investigation of an instance of something in a specific context. Case studies always focus on understanding what is being studied in relation to its environment or context (Flyvbjerg 2011, 301). Also inherent in the idea of a case study is the idea that it represents some wider class of cases, so cases are investigated not simply in their unique particularity but with the thought that studying them will shed light on other cases.

In our case studies, we begin by identifying a particular instance of a practice of moral justification that people are using in an attempt to justify a substantive moral claim about an issue that is under dispute. We try to specify the practice of moral justification that the instance exemplifies by identifying central assumptions people are relying on and their implicit norms of reasoning. We analyze each instance of justification by modeling how it delineates moral domain, characterizes moral agency, specifies reasoning strategies, and prescribes courses of action. The result is a descriptive model of a real-world practice of justification that can be evaluated by our adequacy conditions and compared with the practices other people are using or that philosophers have modeled. Using our adequacy conditions, we assess each practice in order to discern which, if any, is likely to yield morally justified claims in each case. We try to explain why certain practices are exemplary in each case and why others work less well to justify moral claims in each situation. From these explanations, across a range of cases, we develop prescriptive guidelines for when certain practices should be used in certain contexts, and how they should be used in those contexts. The guidelines are ways of linking exemplary practices with their context of use. In order to investigate case studies, philosophers need to know how to select cases, how to specify which features of cases to study, how to evaluate cases, and what to do with the results of these assessments. In what follows we describe in more detail the case study method we recommend as we use it, illustrating its general components.

\subsection{How to Select Cases}

First, our selection of cases is guided by our understanding of what moral justification is as well as gaps we have perceived in philosophical study of moral justification up to this point. Our selection of cases is broad because we select cases that include reasoning practices that have been either ignored or denigrated by previous philosophers, but it is narrowed by our philosophical account of moral justification as a norm-governed practice of exchanging reasons. As regards breadth, our inclusiveness is not random or whimsical but linked with the explicit theoretical goals we are trying to achieve. We tend toward inclusiveness so as to minimize prejudging what may count as moral reasoning. In the case of morality, we regard everyone (almost) as a full-fledged moral agent. If the moral thinking of ordinary people were to be ruled out, this would result in a kind of moral elitism and authoritarianism on the part of philosophers. So we select cases that examine the moral reasoning of people whom contemporary philosophers have tended to exclude, people whose reasoning strategies may be unfamiliar to philosophers or have been denigrated as logical fallacies or as folklore or superstition. Our rationale for tending toward inclusiveness is linked with our adequacy conditions, which express the conviction that 
to be minimally acceptable as candidates for facilitating moral justification, a reasoning practice must be capable of addressing the concerns of those who are most vulnerable in a particular situation and must not too easily rationalize the moral perspectives of those with the most power in that situation. As regards narrowness, this inclusiveness is reined in by our conception of moral justification as involving exchange of reasons, which rules out such practices as reading the runes or reporting visits from the archangels.

Philosophers can further narrow their selection of cases on the basis of what specific question or set of questions they are trying to answer. In our case, we want to understand which practices of moral justification are likely to work best for rationally resolving moral disputes where people are culturally diverse and/or socially unequal. Diversity and inequality are endemic to the world as we know it, and to most situations of intransigent moral disagreement. For this reason we think they should be at the center of philosophical accounts of moral justification, but moral philosophers up to this point have placed them at the periphery, as a problem of application. We place them at the center by selecting cases of moral disagreement in which people have diverse cultural perspectives and/or stand to one another in relations of social power and vulnerability.

Philosophers must further narrow the range of cases they will study under their broad research objectives, for there are likely to be indefinitely many cases that might fit with these broad goals. For example, there are many kinds of diversity and inequality manifest in different contexts and at different periods in history. So just selecting cases of moral dispute marked by diversity and inequality is still too broad. Our goal is not to establish the new, best, single reasoning practice that will be usable to justify moral claims in all situations, or even in all situations of diversity and inequality, but we do want to develop results that are useful for addressing more than one instance of inequality and more than one dimension of diversity. So we select cases that highlight varying types of diversity and inequality, manifest in different arrangements that are still fairly contemporary.

In our own work, we focus specifically on cultural and religious diversity and on power/vulnerability relations linked with gender and global location. Our rationale for focusing on these features of cases is linked with our adequacy conditions and especially our concern to be more inclusive than philosophers have been, but we fully recognize that there are other dimensions of diversity (e.g., political diversity, ethnicity, spiritual [nonreligious] traditions) as well as many other types of social power/vulnerability relations (e.g., class, disability, sexuality, race) that the case studies we investigate do not foreground but that certainly need to be studied. In this article we share a case study of a moral dispute across two communities that are divided by fairly significant cultural differences but linked through a history of colonization.

Our work focuses on disputes from the twentieth and twenty-first centuries. It is highly likely that the epistemic character of moral reasoning has changed with historical shifts that have radically altered the local and global order. We select cases that highlight what we think are particularly salient relations of diversity and inequality in recent history that have been forged through experiences of colonization, democratization, and contemporary globalization so that our guidelines are applicable to occasions of moral justification in the contemporary world. Historical details reaching farther back in time may turn out to be relevant to the analysis of cases, but we fix selection of cases by the time frame of the dispute and we focus on contemporary disputes. For example, the case study we share in this article examines a 
contemporary disagreement over how to morally evaluate practices of female genital cutting among the Maasai.

The central point is that we select cases representing different arrangements of inequality and diversity within a limited, recent historical window so that our results are plausibly applicable for contemporary moral conflicts of this kind and have traction in more than one type of case. We select cases where there are attempts at moral justification, but we broaden our understanding of morality to include more than previous philosophers have included. We narrow our focus further by selecting cases of moral dispute over socially pertinent issues where parties to the dispute are diverse and/or socially unequal. We narrow it further still by specifying which types of diversity and inequality, manifest in which types of arrangements, in which historical time period.

Finally, there is often an element of chance or serendipity in how cases are selected. In our work we select cases from moral disputes we happened to be somewhat familiar with already, either because they were taking place in communities to which we belong or because they were topics in the media and in scholarly debates at the time that were relevant to our current research and teaching opportunities. For example, female genital cutting received extensive attention from the media and from human rights organizations in the last two decades of the twentieth century.

\subsection{Describing, Analyzing, and Evaluating Practices of Moral Justification in Each Case}

Once cases are selected, analyzing and evaluating the practices of moral justification that they exemplify can be divided into five tasks. These are not presented, nor meant to be undertaken, in linear order but are interrelated components of the descriptive, evaluative, and explanatory tasks involved in investigating a case study of moral justification for normative moral epistemology. The five tasks are:

(1) Identify an instance of a moral dispute where there is an attempt at moral justification. On each "side" of the dispute, people are entering a moral claim and giving reasons in support of the claim.

(2) Specify the practice of moral justification that each instance exemplifies. Each instance we observe is an instance of a social practice of moral justification, which is a norm-governed exchange of reasons aimed at moral knowledge. We try to specify the practice as it is being performed in the instance we have observed by identifying the norms governing that process and the assumptions on which it rests.

(3) Analyze each practice by describing how it delineates the moral domain, characterizes agency, specifies reasoning methods and combines them into a strategy, and prescribes action. The result is a descriptive model of a specific practice of moral justification that can be evaluated using our adequacy conditions. Our categories for analyzing real-world practices of moral justification in each case are the same categories we use to analyze practices of justification developed by philosophers. We use these same categories in order to be able to compare real-world practices of justification with those practices defended by philosophers and to evaluate them on the basis of the same adequacy conditions. That is, these categories provide a way of organizing different practices of moral justification into a common rubric for comparison and evaluation.

(4) Evaluate each practice as analyzed. Our adequacy conditions are designed to assess the fit between a practice of moral justification and the context in which it is used, to see which practices are exemplary, which are less capable of yielding moral knowledge in which situations, and why. 
(5) Explain why certain practices appear to be a better fit than others. What is it about the context that makes a certain practice of moral justification a good fit, or not, for that context?

\subsection{Generalizations Across Cases}

In studying each case, we want to understand the relationships between better and worse practices of moral justification that people are using and the situations in which they are using them. Our broader research goal, however, requires providing more than an array of interesting case studies illustrating a range of successful or unsuccessful moral reasoning practices. Recall that the mission we propose for moral epistemology is to develop guidelines for selecting practices of justification appropriate for contexts characterized by cultural and religious diversity and/or pervasive social inequality. Through our best efforts at understanding the relationship between better and worse reasoning practices and the features of the contexts in which they are more successful or less successful, we hope to discover regularities that can be the basis for developing these general normative guidelines. 1

In order to achieve this mission, beginning from a series of case studies, philosophers identify any regularities across cases in order to understand what, if anything, exemplary practices have in common. That is, although the exemplary practices of justification differ across cases (there is a plurality of good practices), we investigate what, if any, features these practices share that make them good fits for situations marked by the kinds of diversity and inequality we study. For example, our larger project highlights three exemplary practices that have been used in three real-world situations marked by three different arrangements of diversity and inequality. We attempt to discern what all three practices have in common that makes them well suited for these types of contexts. These common features are suggestive for helping us understand how to select reasoning practices for these types of situations and how to use them well, and so provide a basis for formulating guidelines.

In the remainder of the article we present one of our case studies in order to illustrate the method of naturalizing that we recommend for moral epistemology. This case study examines a dispute over the moral status of female genital cutting (FGC) as practiced among the Maasai of Kenya and Tanzania. In the next section, we situate the dispute, introduce the disputants, and specify the practice of moral justification each "side" is using in this case. In section 5, we analyze each practice through the categories of domain, agency, reasoning strategies, and outcome, and using our adequacy conditions we evaluate the use of each practice in this situation. We explain why we think that one practice is more successful, that is, more morally rational, than the other in this case although both practices have some weaknesses. We conclude by explaining how, from a series of case studies like this one, philosophers may develop guidelines for selecting justificatory practices appropriate for particular types of social contexts.

\section{A Case Study: Moral Reasoning About FGC Among the Maasai}

\subsection{Situating the Moral Dispute and Introducing the Disputants}

The Maasai live primarily in the Rift Valley region spanning present-day Kenya and Tanzania. $\stackrel{2}{ }$ Maasai "circumcise" both male and female children around puberty and practice what the World Health Organization calls types I and II circumcision of females in a ceremony that is conducted exclusively by elder women. Both male and female rituals mark gendered social transitions. ${ }^{3}$ For females, FGC signifies a transition from being a girl child to becoming a Maasai woman, which is a new social role with new responsibilities. ${ }^{4}$ Becoming a Maasai woman makes one marriageable within the community, and being 
married is a socially necessary condition for being able to build and "own" one's own house within a homestead. Today practices of FGC are illegal in both Kenya and Tanzania where Maasai live, but Maasai are reportedly very resistant to eradication efforts.

Over the past three decades, eradicating FGC has been a high priority on the moral agenda of many women's human rights and development organizations. These organizations argue that FGC is morally wrong and that the international community has a moral responsibility to support, or even spearhead, eradication efforts. We call this influential line of thinking the Women's Human Rights Approach (WHR). WHR has come under criticism from some scholars and activists who study and work in communities where FGC is practiced. $\underline{5}$ Their shared line of critique is that WHR generates misleading, and even morally mistaken, conclusions about why these practices are wrong, who is responsible for the harm, and how to address the harm. These critics offer an alternative moral evaluation of FGC that relies on a different justificatory practice.

This case study examines the two practices of moral justification used in these two contrasting evaluations of FGC. - Both "sides" of this dispute use human rights as moral standards to evaluate FGC among the Maasai. However, each uses a distinct practice of moral justification in which human rights function very differently, generating dissimilar results. The disagreement between the two "sides" of this dispute does not refer to public debate in a single forum with two self-consciously identified opposing viewpoints. Instead, we identify two lines of moral argumentation that have emerged in the global controversy over the moral status of practices of FGC, and we develop a case study that looks at one instantiation of this debate as it has played out in disputes over FGC among the Maasai.? In the remainder of this section, we specify the practice of justification each "side" is using. In section 5 , we analyze and evaluate each practice as it is used in this case.

\subsection{Evaluating Maasai FGC by Appealing to Women's Human Rights}

For the past four decades there has been a global movement to reconceptualize human rights so as to include the often neglected gender-specific rights of women. - Early rights documents, including the Universal Declaration of Human Rights (United Nations 1948), protected individuals against abuse in the public realm of employment and citizenship and from state interference in private affairs, yet they ignored many of the most egregious moral violations against women, which occurred within the socalled private realm of family, religion, and culture. On the early human rights paradigm, many systematic violations against women were defined outside the scope of rights and construed as matters of cultural or religious practice. $\underline{9}$

The Fourth World Conference on Women, held in Beijing in 1995, $\underline{10}$ addressed this issue directly by bringing culture and religion under critical moral scrutiny. This conference produced a document called The Beijing Declaration and Platform for Action, which expands the list of universal rights by offering a gender-specific interpretation of human rights and identifying many social practices that violate these rights, including FGC, which it tendentiously calls FG "Mutilation." says about what it calls FGM: FGM is an act of violence against women that violates their human rights (United Nations $\underline{1995}$, article 113). Violence against women is "any form of gender-based violence that results in or is likely to result in physical, sexual, or psychological harm" (article 113). Violence against women is "a manifestation of the historically unequal power relations between men and women, which have led to domination over and discrimination against women by men and to the prevention of women's full advancement" (article 118). These historically unequal power relations between men and 
women derive "essentially from cultural patterns, in particular the harmful effects of certain traditional or customary practices" (article 118). The Beijing Platform recommends that governments "prohibit female genital mutilation wherever it exists and give vigorous support to efforts among nongovernmental and community organizations and religious institutions to eliminate such practices" (article 232).

The World Health Organization and other U.N. organizations use the gender-specific interpretation of human rights (WHR) articulated in The Beijing Platform to argue that FGC violates the rights of the child and of women "to health, security and physical integrity, and to be free from torture and cruel, inhuman or degrading treatment." $\underline{\text { 12 }}$ The U.N. Office for the Coordination of Humanitarian Affairs has issued at least one report that focuses specifically on Maasai communities (United Nations 2012). This report concludes that Maasai practices of FGC constitute a practice of systematic violence against women that is the result of historical relations of male domination and female subordination that are deeply rooted in Maasai culture. This line of argument is bolstered with accounts of Maasai men and women defending FGC on grounds of respect for culture, which are then used to reinforce the belief that these genderspecific wrongs have cultural roots (United Nations 2012)..$\underline{13}$ The report concludes with the following:

It is the adherence to their own traditions that makes eradication of FGM among the Maasai such an uphill task for those seeking to end the practice. ... Nevertheless, the outside world is slowly influencing the Maasai way of life with more girls and boys being enrolled in formal education and learning about the risks associated with FGM.... The eradication of FGM brings with it the consequence of forever altering the traditions of what is one of the few remaining authentic African societies. ... The challenge anti-FGM campaigners face is how to change this one harmful aspect of Maasai tradition without tainting the authenticity, or undermining the richness, of their culture. (United Nations 2012)

On WHR, the wrong is so-called FGM, characterized as an act of violence against women; the social structures enabling this violence are Maasai gender relations premised on male domination and female subordination, which are a product of Maasai culture; and the agents enacting the violence are Maasai men and complicit elder Maasai women. On this way of framing the issue, it follows that attempts to eliminate this moral wrong must change the culture in which it is embedded.

In order to justify their conclusions, those using WHR advocate a practice of moral justification that appeals to a universal moral standard. We call this practice deductive application of a universal moral standard (DAU). As used in this case by WHR advocates, DAU has at least five central features:

1. DAU appeals to a single moral standard-women's human rights - that is intended to apply to a single phenomenon-FGC-whenever and wherever it occurs, yielding conclusions that are taken to be universal in scope.

2. The moral standard to which DAU appeals is fairly settled. A standard or principle can be more or less abstract. For example, the principle "do no harm" is quite abstract, leaving a lot of interpretive room for deciding what counts as harm. By contrast, women's human rights as articulated in The Beijing Platform are intentionally concrete; they are designed to bring into focus gender-specific wrongs in order to plug the loopholes in earlier conceptualization of human rights. The specificity of a women's human rights standard means that it already contains the core moral analysis. ${ }^{14}$ That is, it defines which social practices are morally wrong and 
provides an account of why they are wrong. This means that anyone using the standard starts his or her moral evaluation with a prefabricated moral frame that foregrounds gender and culture. When the standard is applied in particular cases, other features of the social contexts where practices of FGC occur recede into the background and are obscured from the outset.

3. Relatedly, women's human rights standards have the status of basic principles. Although human rights are not impervious in principle to critique and revision (and although The Beijing Platform document itself demonstrates how they may be revised), they are taken to express fairly widespread, global overlapping consensus on our most reliable moral principles at this point in history. On DAU, "appealing" to women's human rights here means deductively applying a universal standard to concrete cases in order to demonstrate to doubters that particular instantiations of FGC violate women's human rights.

4. It is assumed that further information about the contexts where FGC occurs, including historical information about the emergence and meanings of these practices, has no moral salience. For example, FGC as practiced among the Maasai in Kenya is very different from FGC as practiced among Muslims in Indonesia, but when using DAU a person enters both contexts already knowing that these practices are wrong, and knowing why they are wrong (that is, all instances of FGC reflect and reinforce culturally specific gender relations premised on male domination and female subordination). Contextual and historical details are used to recruit evidence showing how a particular practice is indeed an instance of FGC. On DAU, the person applying the moral standard focuses on the situation to ensure it exemplifies the standard, but not to revise the standard or determine its meaning. DAU recognizes that contextual and historical details may put one in a better position to communicate to resisters the already established true moral conclusions or may be used to make eradication efforts as culturally palpable as possible, but these details do not influence the moral analysis.

5. WHR advocates also use the auxiliary reasoning strategy of imaginative projection to bolster their primary argument by inviting "us" to imagine ourselves in "their" situation of horror. $\frac{15}{\text { One }}$ aim of imaginative projection is to encourage an empathetic response to the situation of victims. As used in this case, inputs to the imaginative exercise include photographs and videos that portray screaming young girls crying uncontrollably. These images are plucked from their context, leaving the imaginer a great deal of latitude to fill in the details.

\subsection{Evaluating Maasai FGC Through the Frame of Colonial History}

Although a WHR approach to FGC is widely accepted, some scholars and activists criticize this argumentative strategy. The critics to whom we refer do not dispute that many practices of FGC are in some sense harmful, nor do they reject women's human rights as important moral tools (Nnaemeka 2005; Nnaemeka and Ezeilo 2005; Abusharaf 2006). Rather, they object to the strategies of moral reasoning used by WHR advocates in their use of human rights, and the way WHR advocates frame the issue and characterize moral agency in evaluating FGC. Taken collectively, the work of these critics offers a sustained argument that WHR systematically conceals features of the social and historical contexts in which FGC occurs that are highly relevant for an adequate moral evaluation of these practices. In this section, we present one version of this criticism and an alternative moral analysis of FGC among the Maasai through the frame of colonial history. We call this a postcolonial analysis approach (PCA).

As mentioned in the introduction to this case, Maasai FGC initiates the social transition of a girl into womanhood. In the period prior to formal colonization in $1921, \underline{\underline{16}}$ social responsibilities associated with 
Maasai womanhood included a significant amount of economic and political authority. Though a married elder male enjoyed political authority over general homestead management, each wife within a homestead built and controlled access to her house (Talle 1998, 133). Anthropologist Aud Talle describes house occupancy as the base of female social agency within Maasai communities $(1998,133)$. For example, Maasai wives had authority to initiate and testify at judicial proceedings, and they participated in dispute resolution both within and between homesteads (Hodgson 1999, 48). Maasai wives controlled the production and distribution of milk, which is a primary food staple for the Maasai; they had to be consulted in decisions about whether to slaughter, trade, or give away an animal; and they were the primary economic agents in a barter economy, traveling to markets outside the homestead in order to barter surplus milk and hides in exchange for other important goods (48).

Talle's research shows that the cultural logic of Maasai life has long linked FGC with economic and political roles for women because only married women could assume these roles and only "circumcised" women could marry. Anthropologist Dorothy Hodgson notes, however, that in precolonial times Maasai gender relations were neither dualistic nor hierarchical but based on notions of complementarity and interdependence. While it was always possible to distinguish between a domestic sphere of home and homestead and a public space outside the homestead, both males and females occupied important social roles in both domestic and public space (Hodgson 1999, 58; Hodgson 2001). Hodgson argues that the term "patriarchy" does not accurately describe the nature of Maasai gender relations prior to colonization because, despite the rigidly defined gender roles for both males and females, social relations between the sexes were not premised on principles of male domination and female subordination.

Maasai gender relations shifted dramatically, however, during and after formal colonization by the British (c. 1920-1961). As Hodgson describes it, British authorities mapped a culturally specific gender ideology onto their perceptions of and interventions into Maasai life, creating "the male domains of public and political in opposition and superior to the female domains of private and domestic" (1999, 57).

There were at least three colonial policies that significantly altered Maasai gender relations. First, the policy of indirect rule required identifying a central Maasai authority to act as an intermediary between the Maasai and the British. In implementing this policy, British authorities assumed that male elders were already "the" political leaders, thereby extending the authority of select male elders over both junior men and women, strengthening and consolidating their power. By creating a centralized male political authority, indirect rule undermined whatever political power Maasai women may have previously enjoyed (Hodgson 1999, 53-55). Second, needing to create a cash economy in order to produce tax revenue for the Crown, the British also transformed a previously female-based barter economy into a newly male-dominated cash economy. Livestock was now to be bought and sold on the market for cash, and colonial authorities assumed that males were the "owners" of cattle. So Maasai men were integrated into the new economy as buyers and sellers of livestock, while Maasai women were dispossessed of their previously shared cattle rights and now struggled to gain access to cash "indirectly through gifts from men or the sale of cattle by their sons or husbands" (57). The third policy, which followed directly from the second, was to implement a new system of taxation. This system designated male elders as "tax payers" and "heads of household," who were now required to pay a "plural wives" tax for "dependent" women living on their homestead (58). 
On the surface, these policies appeared gender-neutral, but they reflected a British gender ideology that was deeply patriarchal. The combined effect of these colonial policies was severe political and economic disempowerment and symbolic devaluation of Maasai women. Foregrounding colonial history reveals that contemporary Maasai gender relations, which today are identified as "authentically" and deeply embedded in Maasai culture, are really a "co-invention" by British colonial authorities and opportunistic Maasai in a fairly recent struggle for power. $\underline{.17}$

The postcolonial analysis (PCA) of Maasai FGC suggests an alternative moral analysis. Contrary to the WHR conclusion, PCA does not portray Maasai FGC as a violent manifestation of the historically unequal power relations between Maasai men and women rooted in inherently patriarchal Maasai cultural patterns. This analysis keeps the idea that the wrong committed is practices of violence against women, and also the idea that the social structures enabling this form of violence include gender relations premised on male domination and female subordination. However, on PCA, the gender relations enabling and maintaining this form of violence result in part from the historical experience of European colonization of Kenya and Tanzania, and so the agents enacting this violence are a complex cast of characters, including British colonizers who imposed a distinctively British patriarchal gender ideology on Maasai through a variety of colonial interventions.

WHR contends that the harmful gender relations supporting Maasai FGC derive from cultural patterns that belong essentially to the Maasai, but PCA argues that this claim is false. Some physical harms or risks associated with Maasai FGC have likely always been present, but PCA suggests that the practice does not become a manifestation of patriarchal violence against women until the colonial encounter, or rather that it becomes a tool of male dominance only in the context of this encounter. The harmful gender relations that today give symbolic meaning to Maasai FGC derive from cultural patterns, but the cultural patterns from which they derive resulted from a forced blending of British gender ideology with preexisting Maasai social categories.

An alternative practice of moral justification supports PCA, although advocates of this approach do not explicitly defend this practice. PCA relies on a practice of moral justification that we call empirically informed reflective equilibrium (EIRE). EIRE, as used in this case, recommends using women's human rights as a universal moral standard that flags morally suspicious gendered practices but interprets the moral standard in light of a particular social and historical context in order to discern whether there is a moral violation in this case, and if so, to generate a comprehensive account of that violation. As used in this case, EIRE has at least five features that distinguish it from DAU used by WHR advocates.

1. On EIRE, moral evaluation of FGC does not start with a single universal moral standard of human rights that already tells us FGC, as a singular phenomenon, is wrong. Instead, EIRE begins with contextual scrutiny of FGC in a particular situation and assumes that there is no culturally neutral description of FGC. Even if physical descriptions of these practices may be similar, their symbolic and cultural meanings vary widely. The "it" being described is not assimilated to practices all over the world. Instead, different cases and their meanings have to be studied in context, which means that EIRE will yield conclusions that are narrow rather than universal in scope. For example, in this case conclusions could only be reached about Maasai FGC. As a practice of reflective equilibrium, EIRE also begins with multiple inputs, including women's human rights, colonial history, and Maasai cultural understandings about FGC. 
2. EIRE accepts the status of women's human rights as important moral principles, which in this case are designed to pick out social practices that manifest violence against women. EIRE notices, however, that despite the attempts to formulate women's human rights in ways that allow little room for interpretation, there are always borderline cases where genital interventions may or may not be argued to be violent (e.g., some symbolic genital nicking). EIRE also notices that cultures are not self-contained, readily identifiable entities, and that there is also not a culturally neutral interpretation of women's human rights.

3. EIRE takes human rights as moral standards that need interpreting in light of particular cases rather than just implemented in a deductive manner. Recall that on the WHR model, which uses DAU, contextual details of particular cases do not influence the moral analysis, because the moral analysis is already given in the standard. By contrast, on PCA, which uses EIRE, contextual details have salience in shaping our moral assessment of the situation and our interpretation of women's human rights and not just in persuading doubters or tailoring eradication efforts. In this case, through reasoning by EIRE, advocates of PCA use historical details to reveal a more comprehensive account of the human rights abuses Maasai women have suffered, which include the abuses they experienced in the colonial period. $\underline{18}$

4. Relatedly, EIRE uses women's human rights as one important source of moral knowledge but appeals to other sources of knowledge-including, in this case, history, gender studies, postcolonial studies, and anthropology-in order to interpret human rights in light of social and cultural contexts, thereby making the reflective equilibrium wide rather than narrow.

5. Finally, imagination is also used in reasoning by EIRE. The imaginative inputs available through EIRE include a rich historical and cultural picture of a particular practice as performed by a specific group of people, which leaves the imaginer with less latitude to fill in details.

\section{Assessing the Alternative Practices of Moral Justification Used in This}

\section{Case}

The practice of moral justification used by WHR is deductive application of a universal moral standard (DAU), and the practice used by PCA is empirically informed reflective equilibrium (EIRE). Both practices use women's human rights as moral standards to evaluate practices of FGC among the Maasai, but the two practices use human rights quite differently. In this section we analyze each practice as used in this case through the categories of moral domain, moral agency, reasoning strategies, and outcome, and we evaluate each practice using the adequacy conditions we have previously defended.

\subsection{Delineating the Moral Domain}

Any practice of moral justification will include some way of framing moral issues that determines which issues fall within the scope of morality and how issues that fall within the frame are characterized. Those using DAU frame FGC as a contemporary violation of a universal human right. DAU diagnoses the violation using a single undifferentiated category of "FG Mutilation," which is defined as a specifically gendered practice in a named but otherwise nonspecific local culture, operating in an unexamined global context. By contrast, advocates of EIRE focus on the specific practice of FGC within the particular ethnic group of the Maasai and analyze this practice in the historical context of European colonization of Kenya and Tanzania in order to discern whether there is a rights violation in this case, and if so, to interpret the violation(s) in light of these details. 
Each way of framing the issue is simultaneously wider and narrower than the other. The DAU frame is broader because, in evaluating this case, it relies on a broad understanding of FGC which it calls FGM, and treats all practices everywhere as morally equivalent. Yet it is also narrower than the EIRE frame because it is preset to foreground gender and culture to the exclusion of geography and history. By contrast, the EIRE frame is narrower than the DAU frame insofar as it resists using a comprehensive category of FGC and instead focuses exclusively on the practice as it occurs among Maasai. Yet, it is also broader and more flexible than the DAU frame insofar as it does not preset the categories of moral evaluation. Instead, it allows salient categories of moral assessment to emerge through empirical inquiry. In this example, those using EIRE place Maasai FGC in the context of more than a century of colonial history. Each delineation of the moral domain highlights and obscures different aspects of the practice of FGC among the Maasai and uses these to justify different moral conclusions.

In this case, we think that the frame employed by those relying on EIRE is a more morally rational way to view the moral domain because this frame makes visible several features of the situation that are morally relevant but are hidden by the DAU framing. Specifically, those employing EIRE are able to show how the social meaning of FGC for the Maasai today has been partially shaped by their experience of colonization, a feature the DAU frame obscures. The moral analysis supported by EIRE is also able to show how disregard of colonial history by using the DAU frame conceals the abuse of global power. Portraying FGC among the Maasai simply as an abusive violation of human rights perpetrated on indigenous women and girls by indigenous men and complicit elder women not only oversimplifies the practice, it also disproportionately brings under moral scrutiny the lives of those with the least global power while simultaneously protecting from moral scrutiny the behavior of those with more global power who have also played a role in this issue. $\underline{\underline{19}}$

Finally, by using a moral frame that foregrounds culture to the exclusion of history and presumes cultures to be self-contained easily identifiable entities, DAU has rationalized eradication efforts premised exclusively on cultural intervention. The colonial experience was already a devastating encounter of violently imposed cultural change. So the DAU frame too easily legitimates potentially harmful and infeasible interventions by people who already have a history of power abuse in this region. By contrast, those using EIRE have the methodological resources to show that this understanding of culture is flawed. Moreover, EIRE encourages moral analysts to be flexible in their framing and to consider a wide range of empirical information pertinent to the particular situation under scrutiny when delineating the moral domain.

\subsection{Characterizing Moral Agents}

The way each practice defines the moral domain shapes the ways in which moral agents are characterized, including those harmed and how they are represented, those who have moral responsibility for the harm, and those who are authorized to evaluate the harm.

How are the victims identified and represented on each approach? By framing FGC exclusively in terms of gender and culture, DAU users take for granted that those wronged are the women who undergo the practice. The women's perspective is assumed through imaginative projection, and they are taken to be exclusively victims rather than agents. DAU does not enable the voices of Maasai women to be heard, or they are quoted from out-of-context interviews where they are cited as defending FGC on cultural 
grounds. These quotes get recruited as evidence to show that Maasai women are prisoners of either false consciousness or moral backwardness.

By contrast, the broader frame of colonial history enables those using EIRE to notice that all Maasai, men and women, have been harmed by the imposition of British gender ideology onto Maasai social life while simultaneously allowing them to explain why and how Maasai women have suffered genderspecific injury. Those using EIRE in this case do not say much beyond this about the women who undergo FGC, nor do they include their voices, which is a significant limitation of the way this practice is used in this case. EIRE does, however, leave open the possibility of perceiving that Maasai women might "choose" the practice in some sense and that they are not necessarily always being dragged kicking and screaming. Moreover, those using EIRE can incorporate historical facts that may suggest an interpretation of contemporary Maasai resistance to eradication projects as expressing moral agency rather than as necessarily revealing false consciousness or moral backwardness. For example, EIRE enables its users to notice the moral relevance of a 1950s Kenyan movement, in which Maasai participated, called "I Will Circumcise Myself," whereby young girls performed genital cuttings on themselves in order to defy the legal ban imposed by British colonial authorities (Thomas 1996). EIRE enables its users to consider the possibility that Maasai resistance to contemporary eradication efforts reflects suspicion about foreign-led interventions premised on cultural change by people from global powers who have a morally poor track record in this region. By contrast, DAU makes it very difficult to consider this possibility and instead encourages viewing Maasai women as mere objects of moral concern. So despite its limitations as used in this case, EIRE makes it possible for its users to take a more nuanced perspective on moral agency than DAU does because EIRE encourages the seeking of empirical evidence that is morally relevant to the victimhood or agency of Maasai women.

Who is assigned moral culpability on each strategy? DAU encourages its users to blame people who currently promote this FGC practice, either directly or indirectly, whereas EIRE enables consideration of the culpability of a much broader range of candidates, including British colonizers who are now dead. Those using the DAU frame identify members of the Maasai community exclusively as defendants and also as the only defendants in this case. People located in the global north are methodologically excluded as having no direct involvement with the practice, a distance that suggests that their proper roles in its moral evaluation are those of prosecutor and impartial moral judge. By contrast, EIRE allows its users to position people located in the global north as possible parties to the harm and so as defendants in this case, thereby suggesting that the Maasai are not the only defendants. And as just described, EIRE also enables repositioning all Maasai, men and women, as legitimate plaintiffs in a more complex case of human rights abuse, which includes the abuse of colonization, thereby suggesting that Maasai are not exclusively defendants.

Who is likely to be assigned moral standing to evaluate the harm on each approach? With DAU, human rights are taken as universal standards of moral evaluation that apply to everyone everywhere in the world and in whose maintenance everyone has a stake simply by virtue of being human. As with all human rights problems, the state is primarily responsible to address the harm, and indeed Kenya recently outlawed all participation in FGC in any way (Boseley 2011). However, DAU does not preclude assuming that everyone everywhere has at least a prima facie claim to participate equally in evaluating human rights abuses, an assumption that opens the possibility of legitimating NGO interventions and perhaps even state support for religious organizations seeking to intervene. Although EIRE does not preclude assuming that people everywhere have a stake in eliminating rights violations, this justificatory 
practice does not require assuming that everyone's stake in every case is the same. In this example, by focusing more narrowly on Maasai FGC, this approach implicitly identifies central protagonists, including Maasai men, Maasai women, British colonial authorities, contemporary governments attempting to outlaw FGC, and development organizations promoting eradication efforts in Maasai communities. It simultaneously places others as peripheral stakeholders in this case, including people living in other communities where FGC is practiced, such as Muslims in Indonesia, as well as people in parts of the world whose connection with Maasai and their recent history is remote or nonexistent.

We think that in the particular case of FGC among the Maasai, EIRE enables a more acute assessment of moral agency, including moral standing and responsibility, than DAU allows. EIRE permits consideration of the possibility that DAU may enable the abuse of social power insofar as positioning the north as the external moral critic unfairly makes people in this global location judge and jury of their own case. EIRE also makes it possible for users to consider that Maasai opposition to eradication efforts may not be matters of moral backwardness, ignorance, or false consciousness but instead may reflect reasonable skepticism about interventions promoting cultural change. Finally, EIRE allows for a more variegated and fairer picture of who has legitimate standing to evaluate the harm.

\subsection{Strategies of Moral Reasoning}

In DAU argumentation, top-down inferences run in one direction from general to particular. The argument is bare of details; most contextual factors are taken not to be morally salient, and might even be distracting. $\underline{20}$ DAU bolsters argumentation through a form of imaginative projection.

By contrast, EIRE involves reasoning by reflective analysis of particular cases in light of universal standards and reinterpretation of universal standards in light of particular cases. EIRE also permits seeking out the perspective of those who have the most at stake in the issue under scrutiny and who are the most epistemically disadvantaged. Those using the EIRE argumentative strategy seek reflective equilibrium among various descriptions of "the" problem, various accounts of the salience and interpretation of proposed moral standards, and a variety of contextual factors, including historical and power dimensions. A "horizontal" web of inferences runs among these.

In this case, our success criteria favor the reasoning recommended by EIRE as more rational than the reasoning recommended by DAU. EIRE's flexibility and relative openness to new interpretations makes it less likely than DAU to enable legitimation of power abuse and more likely to generate reasoning that is plausible, is usable, and yields feasible conclusions. Human rights are a powerful and important moral tool, but in this case they cannot function as the only moral tool. Moreover, they cannot be used rationally in this type of context without recognizing that the meaning of human rights standards must be interpreted in each case, not just preformed or implemented; furthermore, the interpretations must be plausible to those whose lives stand to be most disrupted, so that the conclusions have normative force and are feasible for them. In some contexts, deductive application of a universal moral standard might be morally rational and might yield credible moral conclusions. Using EIRE, however, we can see that the use of the DAU reasoning in the case of the Maasai allows for a corrupt moral analysis of FGC, and that the reasoning strategy of DAU is itself corrupt in this case.

Moreover, EIRE also uses the imagination to encourage empathetic response, but it avoids sensationalizing and "othering" the "exotic" and "savage" social practices of "darkest Africa." EIRE provides a more accurate cultural and historical picture of FGC among the Maasai, leaving the imaginer 
with less latitude to fill in the details and directing the imaginer to cultivate a more accurate empathetic response, which includes a more informed understanding of the practice as it occurs in this community, a more complete picture of who has been involved in establishing the salience of the practice today, and self-knowledge about why Maasai might receive proposed interventions from members of Western nations with suspicion. By contrast, the imaginative inputs available on DAU are decontextualized photographs and video clips. The imaginer fills in the details, which makes it too easy to rely on stereotypical information about unfamiliar peoples in "exotic" faraway places, and thereby too easy to generate an inaccurate empathetic response that enables power abuse.

For all of these reasons, we argue that EIRE is capable of delivering a more comprehensive account of the moral harm, a fairer account of who is responsible for the harm, and a more morally defensible basis for decisions about whether to intervene, and if so who should intervene, and how.

\subsection{Courses of Action Prescribed}

Those who reason according to WHR prescribe state intervention to prohibit practices of FGC and recommend institutional support to NGOs and religious organizations on grounds of humanitarian intervention. The courses of action they prescribe advocate cultural change, although they puzzlingly require that somehow intervening agencies must change just a single aspect of Maasai culture without damaging the supposed authenticity or richness of Maasai traditions. In this case, those using EIRE do not prescribe a determinate course of action for Maasai, which is a limitation of the way the practice is used in this case. However, EIRE's requirement of attention to the role of contextual details in moral analyses means that this approach encourages users to prescribe responses on a case-by-case basis, and in addition leaves open the possibility that in some cases a response may not be warranted. And when a response is warranted, the reasoning strategy of EIRE is more likely to yield a course of action tailored appropriately to the material and existential realities of the people who have the most at stake in the issue. Using EIRE, they are more likely to attend to historical and present power relations between those proposing a course of action and those to whom they make such a proposal. For example, those using EIRE might prescribe something like symbolic genital nicking or circumcision-with-words ceremonies, which have emerged in other communities with similar histories. These responses are designed to mitigate the long-term health consequences and social harms associated with these practices, while preserving elements of their cultural and social significance. So although DAU delivers a determinate conclusion in this case, our success criteria suggest that those using EIRE are more likely than those using DAU to reach moral conclusions that are less susceptible to power abuse and more feasible for those most directly affected.

\subsection{Summing Up Our Assessment of These Practices of Moral Justification}

We conclude this section by drawing explicitly on our four assessment conditions to summarize our evaluation of DAU and EIRE as practices of moral justification in this case. Although there may be other situations in which DAU works well, we conclude that DAU is a less fitting practice of moral justification than EIRE is in this particular case.

Plausibility. DAU and EIRE both appeal to human rights, but in our view EIRE enables a more plausible way of using human rights as tools of moral assessment than DAU does in this case. DAU assumes that "the" answer to "the" problem exists already in the form of a universal principle of women's human rights that is already formulated at the appropriate level of abstraction for application in all contexts; it is not too thin and not too thick. By contrast, EIRE assumes that the relevant standard or principle and 
the appropriate level of interpretation for it will emerge as the "wrong" comes into clearer focus. Interpreting human rights in light of the details of a particular practice of FGC in a specific place is more likely to be able to link the moral authority of the conclusion with the reasoning that generates that conclusion. Users of EIRE in this case also provide a more plausible story about the variety of rights violations involved in this case and a more plausible interpretation of those violations that is likely to seem less arbitrary or baffling to those most directly impacted.

Usability. Usability requires that all those affected be able to participate in moral reasoning; this may require, for example, that participants utilize particular rituals or forms of speech. In this case, Maasai are not actually using either reasoning strategy, which is a significant weakness of both approaches. However, in our view EIRE has greater potential than DAU to better satisfy the usability condition in situations like this one. The DAU strategy makes no room for cultural standards or contextual details to interpret the meaning of rights; they are used only to tailor eradication efforts. Moreover, Maasai opposition to moral arguments supported by DAU reasoning suggests that the reasoning being offered is not plausible to many Maasai. One reason for this may be that the DAU reasoning strategy is not usable by them in the sense of broadly conforming to internal cultural standards. EIRE leaves open the possibility for Maasai standards of justification to be used because the reasoning strategy of EIRE encourages its users to continually seek out new information from the particular social context under scrutiny, and so it can incorporate Maasai moral perspectives in order to interpret, revise, or broaden human rights principles.

Abuse of power or vulnerability. In our view, DAU is more vulnerable to abuse of power and vulnerability than EIRE is in this case. DAU as used in this case brings under moral scrutiny the least powerful while shielding the most powerful from scrutiny. DAU also makes its own moral framework appear incontrovertible while too easily lending itself to discrediting anyone who uses an alternative framework. In our assessment, DAU is more susceptible to power abuse in this context for at least three reasons. First, it relies on a static and oversimplified notion of culture in a context in which cultural interventions are not innocent but track global power relations both historically and at present. Second, it also tends to assign "culture" to those with the least global power while simultaneously making dominant global perspectives appear cultureless, which they are not. Finally, DAU foregrounds gender $\underline{21}$ but places gender relations in a historical vacuum, and so obscures the complex web of historical interactions among nations and cultures that have produced the specific gender relations within Maasai communities that are today the object of global moral criticism. The epistemic stance enabled by DAU is eerily similar to the perspective of British colonizers who perceived gender and culture in the Maasai practices that were exotic or foreign to them, but who failed to see their own policies as gendered and instead regarded them as natural and invisible. Although EIRE is certainly not immune from being abused, it enables corrigibility and discourages dogmatism. EIRE's insistence on seeking out empirical information relevant to understanding the contemporary social meaning of Maasai FGC and the conditions that enable its continuance, as well as EIRE's ability to incorporate Maasai perspectives, make this reasoning strategy less susceptible to power abuse than DAU in this situation.

Feasibility. Maasai are reported as very resistant to eradication efforts justified using DAU, efforts that include state prohibition of FGC coupled with NGO and religious organization interventions that advocate cultural change. In our view, this resistance provides prima facie evidence that the moral conclusions defended by DAU are not feasible to many Maasai. The conclusions may seem arbitrary, baffling, or suspicious given previously devastating interventions premised on cultural change. The interventions might also be materially or existentially impossible for many Maasai, given the real limitations of their situation and the complicated links between Maasai FGC and social life. In our view, 
EIRE is likely more capable of delivering more feasible recommendations than DAU because people using EIRE are not committed to prejudging cases but instead are encouraged to seek out all morally salient information, including alternative moral perspectives. This means that EIRE-derived solutions are likely to be premised on more nuanced assessments of the risks and benefits of eradication efforts in particular situations and to find solutions that are more likely to be real possibilities for people in the contexts they actually live in.

\section{Conclusion}

Although in this case EIRE is a more successful reasoning model than DAU, using EIRE does not guarantee a correct moral analysis for all cases. The upshot of our case study is not to defend EIRE as the new privileged supermodel for all contexts. Certain aspects of EIRE are likely good reasoning practices in other contexts too, but in other contexts the most rational practice of moral justification might look very different. We are not defending any single procedure as the best for all contexts, or even for all contexts of diversity and inequality. Rather, through a series of case studies like this one, we are looking for regularities across cases in reasoning strategies that are more and less successful. For example, one regularity might be that in situations of moral controversy where people are culturally diverse and socially unequal, deductive application of a universal moral standard is inadequate because it proves to be too susceptible to misuse by those with more social power. In other types of situations where interlocutors share greater cultural competence and similar histories, and where the power inequalities are less disparate, deductive application of a universal standard might be morally rational.

To conclude we return once more to the analogy with clinical research. There are enough physiological similarities among human beings to predict that similarly situated human beings will respond similarly to a drug protocol. Yet vast differences in cultural, political, and environmental aspects of social life situate people differently enough to determine the effectiveness of a drug protocol only partially, meaning that researchers often cannot proclaim a drug effective for all contexts. They have to study the relationship between a drug protocol and its context of use to see if in fact it will be effective in that context.

Similarly, we have argued that a variety of social and cultural factors partially determine the fittingness of a moral reasoning practice for a particular context, meaning that the mission to discover a single superstrategy that can justify moral claims in any and all contexts may be a mission impossible. Instead, we propose that philosophers study the interaction between reasoning practices and their context of use in order to judge which practices are likely capable of justifying moral claims in particular types of contexts, and to understand why-what it is about the context that makes some reasoning practices effective and others ineffective or even harmful. From a series of cases, philosophers may discover regularities that can be the basis for developing normative guidelines for selecting reasoning practices likely to be appropriate for different types of situations, which is our newly stated mission for moral epistemology.

\section{Footnotes}

1 We do not take up this part of the case study method in this essay.

2 The Maasai are pastoralists, although maintaining this seminomadic lifestyle is increasingly difficult because state and private "ownership" of land forces them to acknowledge nation-state borders between Kenya and Tanzania. Maasai communities are organized around homesteads and are 
traditionally polygamous. Gender and age are primary categories of social organization within Maasai communities.

3 Male circumcision is not as much challenged by outsiders as FGC, on grounds that male circumcision is not comparable in its harmful physical and sexual consequences to practices of FGC. It is at least arguable, however, that male circumcision does not draw moral outrage from members of Western nations because "we" routinely practice it, at least in the United States (much less so in the United Kingdom), and so it does not appear morally problematic.

4 One Maasai woman interviewed by anthropologist Barbara Hoffman remarks that a female person who has not experienced FGC is still considered a girl, even if she has had ten children. See Hoffman 2002.

5 These critics include scholars who have training in African history, gender studies, anthropology, and postcolonial studies, and activists who have worked to address a variety of women's issues, including FGC, in local communities. See Abusharaf 2006. See also Nnaemeka 2005. We have discerned a shared line of critique and alternative justificatory strategies used to evaluate FGC among these thinkers and activists.

6 This case study draws from a less developed version by Tobin published elsewhere. See Tobin 2009 . Although the basic details of the case are the same, here we use the case study to develop several epistemological and methodological points not made in the earlier work.

7 Another prominent strategy of moral opposition to Maasai FGC emphasizes the health risks and physical and sexual harms associated with these practices. The health-based approach has led to modifications in how FGC is administered to make it safer and more consensual, but this argument strategy does not directly attack the symbolic meanings of FGC or the cultural and social structures that support their continuation. Our case study focuses instead on two lines of moral argumentation that emphasize violence against women as the objectionable feature of Maasai FGC.

8 This movement has been articulated in the United Nations' series of international conferences on women: World Conference of the International Women's Year (Mexico City, 1975), reports available at http://www.un.org/womenwatch/daw/beijing/mexico.html; World Conference of the United Nations Decade for Women: Equality, Development and Peace (Copenhagan, 1980), reports available at http://www.un.org/womenwatch/daw/beijing/copenhagen.html; World Conference to Review and Appraise the Achievements of the United Nations Decade for Women: Equality, Development and Peace (Nairobi, 1985), reports available at http://www.un.org/womenwatch/daw/beijing/nairobi.html; Fourth World Conference on Women: Action for Equality, Development and Peace (Beijing, 1995), reports available at http://www.un.org/womenwatch/daw/beijing/fwcwn.html. Perhaps the most prominent outcome of these conferences was the Convention on the Elimination of All Forms of Discrimination Against Women (CEDAW), initiated by the First World Conference (1975) and prepared by groups within the 1976 Commission and Third Committee of the General Assembly from 1977 to 1979 for presentation at the Second World Conference (1980). See United Nations, "Convention on the Elimination of All Forms of Discrimination Against Women (CEDAW)," adopted by the U.N. General Assembly (1979), available at http://www.un.org/womenwatch/daw/cedaw/text/econvention.htm. Since the Four World Conferences, Review and Appraisal sessions have been held every five years (2000, 2005, 2010). Critics identified that the problem was not just discriminatory application of human rights 
standards but was rooted in a narrow and gender-biased conceptualization of human rights, which covertly assumed that the bearer of rights was gendered masculine.

9 Susan Okin discusses the example of slavery being considered a human rights violation but the practice of bride selling being perceived as a protected cultural practice rather than an instance of slavery. See Okin 2000, 29.

10 See our footnote 8.

11 The Beijing Platform works within a human rights framework that builds on CEDAW by giving a gender-sensitive interpretation of human rights. It details several strategic objectives and recommendations for government action in order to implement these objectives. It is housed under and used by the United Nations Entity for Gender Equality and the Empowerment of Women (U.N. Women) and the United Nations Development Fund for Women (UNIFEM).

12 See World Health Organization 2012. The World Health Organization's "fact sheet" on FGM states that "FGM is recognized internationally as a violation of the human rights of girls and women," reflecting "deep-rooted inequality between the sexes constituting an extreme form of discrimination against women" that violates their rights. Another U.N. publication (United Nations 2012) explains the cultural roots of FGM in several tribal populations living in Kenya, and then advocates in favor of the international response, which recognizes FGM as "a violation of the fundamental human rights of girls and women." In 2008, the United Nations designated February 8 as "International Day of Zero Tolerance of FGM."

13 Maasai who were asked why the spiritual and educational aspect of FGM ceremonies could not be isolated from the physical cutting are reported as responding with "a seemingly unchallengeable argument: 'It is our tradition-we must follow our culture'" (United Nations 2005).

14 Indeed, labeling them as "mutilation" is obviously a thick interpretation.

15 This invitation to imagine is encouraged through appeals to emotion-for example, using morally charged language such as "mutilation" and provocative imagery to promote disgust, compassion, and anger. Sometimes media are used to assist people in this imaginative feat by providing videos that show these practices or reenactments of them in which young girls are heard screaming in pain or crying uncontrollably. See Walker and Parmar 1993. See also United Nations 2012, which provides links to a documentary bearing the name of the report, "Razor's Edge," and uses visual and auditory means to facilitate the imaginative feat.

16 Formal colonization was initiated in 1921, but the British began forcibly removing Maasai from their land c. 1904 and again in 1911.

17 Hodgson's account does not romanticize or oversimplify the role of Maasai men in this process. Opportunistic Maasai took advantage of colonial policies in order to strengthen their political and economic power over both other lower-status men and women (1999, 64-65). Uma Narayan (1997, 81-118) makes a similar point regarding disputes over the moral status of sati and dowry-related deaths in India. Through careful historical analysis (in particular, through a frame of colonial history), Narayan shows how the practice of sati becomes an Indian "tradition" through struggles for power between some Indian men and British colonial authorities.

18 One way to characterize the contrast between the two reasoning practices is that whereas DAU makes the picture (that is, a particular instance of FGC) fit a preset frame, EIRE tailors the frame to fit a picture of the practice that comes into clearer focus with attention to more contextual details. 
19 For example, it is at least arguable that other genital cuttings such as male circumcision deserve comparable moral attention, or that other issues such as "acid scarring," where acid is thrown on women's faces to disfigure them for life, deserve at least equally wide-scale global opposition campaigns. But these practices are prevalent in countries with greater global power than Kenya and Tanzania, and so it is at least arguable that they get less moral attention because the places where they occur are more impervious to moral scrutiny.

20 Interestingly, the reasoning strategies used by scholars and activists to develop the women's human rights framework articulated in The Beijing Platform resembles empirically informed reflective equilibrium. Human rights were taken as important moral standards but then interpreted in light of the specific moral experiences of women in a variety of cultural contexts, revealing a need to radically reconceptualize rights in order to address women's rights violations. Yet once WHR had received relatively secure status as a set of internationally recognized moral standards, it became easy for members of more powerful global communities to revert to deductive application of these standards. In some circumstances this might be an appropriate reasoning strategy, but in general when moral disputes involve interlocutors from former colonial powers and contemporary global powers with histories of colonial interventions in those regions, deductive application of rights standards is likely to be an inadequate justificatory strategy because it is too susceptible to misuse by those with more global power.

21 The WHR approach has made visible gender power abuse across cultural and national contexts, which had been systematically concealed by previous human rights frameworks, and to the extent it has done this it has been successful.

\section{References}

Abusharaf, Rogaia Mustafa. 2006. Female Circumcision: Multicultural Perspectives. Philadelphia: University of Pennsylvania Press.

Boseley, Sarah. 2011. "FGM: Kenya Acts Against Unkindest Cut." The Guardian (September 8). Available at http://www.guardian.co.uk/society/sarah-boseley-global-health/2011/sep/08/womenafrica?INTCMP=SRCH

Flyvbjerg, Bent. 2011. "Case Study." In The Sage Handbook of Qualitative Research, fourth edition, edited by Norman K. Denzin and Yvonna S. Lincoln , 301-316. Thousand Oaks, Calif.: Sage.

Hodgson, Dorothy L. 1999. "Pastoralism, Patriarchy, and History: Changing Gender Relations Among Maasai in Tanganyike, 1890-1940." Journal of African History 40:41-65.

Hodgson, Dorothy L.. 2001. " 'Once Intrepid Warriors': Modernity and the Production of Maasai Masculinities." In Gendered Modernities: Ethnographic Perspectives, edited by Dorothy L. Hodgson , 105-145. New York: Palgrave Macmillan.

Hoffman, Barbara. 2002. "Womanhood and Circumcision: Three Maasai Women Have Their Say" (DVD). Berkeley: Berkeley Media.

Narayan, Uma. 1997. Dislocating Cultures: Identities, Traditions, and Third-World Feminism. New York: Routledge.

Nnaemeka, Obioma. 2005. Female Circumcision and the Politics of Knowledge: African Women in Imperialist Discourses. Westport, Conn.: Praeger.

Nnaemeka, Obioma, and Joy Ngozi Ezeilo (eds.). 2005. Engendering Human Rights: Cultural and Socioeconomic Realities in Africa. New York: Palgrave Macmillan.

Okin, Susan M. 2000. "Feminism, Women's Human Rights, and Cultural Differences." In Decentering the Center: Philosophy for a Multicultural, Postcolonial, and Feminist World, edited by Uma Narayan and Sandra Harding , 26-46. Bloomington: Indiana University Press. 
Talle, Aud. 1998. "Female and Male in Maasai Life: Aging and Fertility." In The Politics of Age and Gerontocracy in Africa: Ethnographies of the Past and Memories of the Present, edited by Mario I. Aguilar , 125-150. Trenton, N.J.: Africa World Press.

Thomas, Lynn M. 1996. "'Ngaitana (I Will Circumcise Myself)': The Gender and Generational Politics of the 1956 Ban on Clitoridectomy in Meru, Kenya." Gender and History 8:338-363.

Tobin, Theresa. 2009. "Globalizing Feminist Methodology: Building on Schwartzman's Challenging Liberalism." Hypatia 24, no. 4:145-164.

United Nations. 1948. Universal Declaration of Human Rights. Available at http://www.un.org/en/documents/udhr/

United Nations. 1995. The Beijing Declaration and Platform for Action: Report of the Fourth World Conference on Women. Available at http://www.un.org/esa/gopher-data/conf/fwcw/off/a20.en

United Nations. 2005. "Kenya: Justifying Tradition: Why Some Kenyan Men Favour FGM." Available at http://www.irinnews.org/Report/53322/KENYA-Justifying-tradition-why-some-Kenyan-menfavour-FGM

United Nations. 2012. "In Depth: Razor's Edge-The Controversy of Female Genital Mutilation/Africa: When Culture Harms the Girls: The Globalization of Female Genital Mutilation." Available at http://www.irinnews.org/IndepthMain.aspx?reportid=62462\&indepthid=15

Walker, Alice, and Pratibha Parmar. 1993. Warrior Marks (film). Available at http://www.wmm.com/filmcatalog/pages/c49.shtml

World Health Organization. 2012. "Female Genital Mutilation: Fact Sheet." Available at http://www.who.int/mediacentre/factsheets/fs241/en/index.html 